\title{
Platelet reactivity in diabetic patients with invasive Klebsiella pneumoniae liver abscess syndrome
}

\author{
Chen-Hsiang Lee ${ }^{1,2}$ \\ Seng-Kee Chuah ${ }^{2,3}$ \\ Wei-Chen Tai ${ }^{2,3}$ \\ I-Ling Chen ${ }^{4}$
}

'Department of Internal Medicine, Division of Infectious Diseases, Kaohsiung Chang Gung Memorial Hospital, Kaohsiung, Taiwan; ${ }^{2}$ Chang Gung University College of Medicine, Kaohsiung, Taiwan; ${ }^{3}$ Department of Internal Medicine, Division of Gastroenterology, Kaohsiung Chang Gung Memorial Hospital, Kaohsiung, Taiwan; ${ }^{4}$ Department of Pharmacology, Kaohsiung Chang Gung Memorial Hospital, Kaohsiung, Taiwan
Correspondence: Chen-Hsiang Lee Division of Infectious Diseases, Kaohsiung Chang Gung Memorial Hospital, 123 Ta-Pai Road, Naso-Sung District, Kaohsiung City 833, Taiwan Tel +88677317123 ext 8304 Fax +88677324804 Email lee900@adm.cgmh.org.tw
This article was published in the following Dove Press journal: Infection and Drug Resistance

\begin{abstract}
Objective: Platelets catalyze the development of hyperinflammation and microthrombosis and contribute to increases in accumulation of circulating platelet-leukocyte complex, the key event in the development of disseminated infection.
\end{abstract}

Subjects and methods: To determine the relationships of platelet activity in diabetic patients with invasive Klebsiella pneumoniae liver abscess syndrome (IKLAS), a total of 175 diabetic patients with community-acquired Klebsiella pneumoniae (KP) bacteremia were included in this study. We compared the platelet reactivity of 40 patients with IKLAS, 40 patients with nonIKLAS, and eight healthy controls using a whole-blood flow cytometry-based assay.

Results: Patients who were infected with strains expressing $\mathrm{K}_{1} / \mathrm{K}_{2}$ serotype (adjusted odds ratio [AOR], 8.81; 95\% CI, 2.18-35.53) and those with $\mathrm{HbA}_{1 \mathrm{c}} \geq 9 \%$ (AOR, 4.97; 95\% CI, 1.73-14.23) were more likely to present with IKLAS, whereas those who had recent therapy with aspirin (AOR, 0.17 ; 95\% CI, 0.04-0.79) were less likely to present with IKLAS. Among patients with IKLAS, patients with a poor glycemic control were more likely to present with hepatic venous thrombophlebitis than those with suboptimal or good glycemic control $(P=0.03)$. Patients with IKLAS had a higher median fluorescence intensity of the platelet membrane expression of $\mathrm{P}$-selectin than those with non-IKLAS ( 78.0 vs $28.0, P<0.001)$ and controls $(78.0$ vs $22.0, P<0.001)$. The IKLAS group also demonstrated a significantly higher platelet-monocyte aggregation and higher

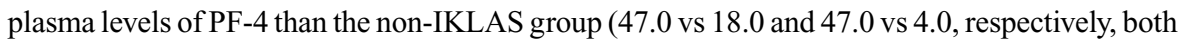
$P<0.001$ ) and controls (46.0 vs 24.0 and 46.0 vs 13.0 , respectively, both $P<0.001$ ).

Conclusion: Diabetic patients with IKLAS demonstrated platelet hyperreactivity, which may be associated with a higher risk for vascular complications.

Keywords: Bacteremia, Glycated hemoglobin, vascular complications, Thrombophlebitis, Aspirin

\section{Introduction}

Klebsiella pneumoniae (KP) is a common cause of community-acquired or hospitalacquired infections such as urinary tract infections and pneumonia. A clinical manifestation of KP infection known as invasive Klebsiella pneumoniae liver abscess syndrome (IKLAS), defined as liver abscess with contemporaneous metastatic KP infections at other body sites, has been increasingly reported, ${ }^{1-3}$ particularly in Asia for decades ${ }^{4}$ and recently worldwide. ${ }^{2,3}$ IKLAS typically infects patients with diabetes mellitus (DM) ${ }^{1,4}$ causing various metastatic infections such as meningitis, endophthalmitis, lung abscess, or fasciitis. ${ }^{4,5}$

$\mathrm{KP}$ is classically defined as an extracellular pathogen. We recently reported that the serotype $\mathrm{K} 1$ of this species, the causative agent of IKLAS, could survive 
within neutrophils and trigger the delay in apoptosis. ${ }^{6} \mathrm{We}$ suggested that the KP serotype K1 can extend the lifespan of neutrophils, making them suitable host cells for bacterial survival and multiplication after infection occurs. This organism may use neutrophils as "Trojan horses" for subsequent infection of other cells. Hence, further studies are needed to identify the factors other than neutrophils that can trigger the development of disseminated infections due to KP.

Previous studies revealed that Staphylococcus aureus or Salmonella spp. can release toxins and/or use surface proteins that subsequently bind to platelet receptor, which can lead to platelet hyperreactivity and contribute to a higher risk for vascular complications. ${ }^{7}$ In patients with invasive $\mathrm{KP}$ infection, the number of thrombotic complications is striking. ${ }^{8}$ Although the essential role of platelets is to maintain vascular integrity and contribute to homeostasis, platelets can also contribute to the innate immune response. ${ }^{9}$ These platelet-leukocyte aggregates have the capacity to act as circulating platforms for generation of a thrombus, producing clots that may lodge in the microvasculature, obstructing the blood flow, and leading to dissemination. Thus, this study aimed to describe the risk factors of IKLAS among DM patients with community-acquired KP bacteremia. In addition, we compared the features of computed tomography $(\mathrm{CT})$ of the abdomen of the patients with IKLAS to ascertain possible predictors of IKLAS among DM patients with different glycemic control. We also studied the platelet reactivity, platelet-monocyte aggregate (PMA), and activation of plasmatic coagulation in a cohort of diabetic patients with KP bacteremia who had either IKLAS or non-IKLAS.

\section{Subjects and methods Study design and participants}

DM patients who were admitted to Kaohsiung Chang Gung Memorial Hospital (KCGMH) between January 1, 2013, and December 31, 2016, with community-acquired monomicrobial bacteremia caused by KP were enrolled in this study to investigate the risk factors of IKLAS. Only the KP isolates from the patients' first blood collection were used in this study. Peripheral blood samples were collected for the whole-blood flow cytometry-based assay as described later from eight healthy male volunteers aged between 25 and 40 years and 80 of the participants after KP bacteremia was confirmed randomly. All protocols used in the present study have been approved by the institutional review board of KCGMH (201601500B0). The patients were made anonymous to maintain confidentiality of the data. All participants including eight healthy male volunteers provided their written informed consent to participate in the study.

The diagnosis of IKLAS was made in patients with liver abscess who developed contemporaneous metastatic $\mathrm{KP}$ infections at other body sites such as renal abscess, splenic abscess, meningitis, empyema, mycotic aneurysm, necrotizing fasciitis, or endophthalmitis. ${ }^{10}$ To investigate the predisposing factors of IKLAS, the following clinical variables were assessed: age, sex, comorbidities (including cardiovascular diseases, liver cirrhosis, malignancy, chronic renal failure, alcoholism, and biliary tract disease), and a history of receiving high-dose steroids ( $>20 \mathrm{mg}$ of prednisolone daily for $>3$ weeks) or aspirin in the previous month prior to the collection of their first KP-positive blood culture. To assess the glycemic control in these DM patients, the $\mathrm{HbA}_{1 \mathrm{c}}$ was determined. The impact of glycemic control on various outcomes of interest was evaluated by grouping all patients into three categories on the basis of $\mathrm{HbA}_{1 \mathrm{c}}$ levels: $\mathrm{HbA}_{1 \mathrm{c}} \leq 7 \%$ (good glycemic control), $\mathrm{HbA}_{1 \mathrm{c}}$ 7\%-9\% (suboptimal glycemic control), and $\mathrm{HbA}_{1 \mathrm{c}} \geq 9 \%$ (poor glycemic control). ${ }^{11}$

\section{Bacterial isolates and serotype determination}

All KP isolates were identified using standard methods. Capsular genotyping of seven clinically significant capsular types $\left(\mathrm{K}_{1}, \mathrm{~K}_{2}, \mathrm{~K}_{5}, \mathrm{~K}_{20}, \mathrm{~K}_{54}, \mathrm{~K}_{57}\right.$, and $\left.\mathrm{K}_{\mathrm{N} 1}\right)$ was performed with the use of PCR assay. Briefly, PCR was performed using primers designed for the $c p s$ variable region. Genomic DNA was extracted by boiling selected colonies in distilled water for 10 minutes. The PCR conditions were $96^{\circ} \mathrm{C}$ for 3 minutes, followed by 30 temperature cycles of $96^{\circ} \mathrm{C}$ for 30 seconds, $53^{\circ} \mathrm{C}$ for 15 seconds, and $72^{\circ} \mathrm{C}$ for 30 seconds. ${ }^{12}$

\section{Characteristics of clinical imaging}

All patients with IKLAS underwent contrast-enhanced CT of the abdomen. In some patients, CT studies were performed during follow-up to monitor complications. We only reviewed the first imaging studies. The following features were recorded: 1) number of abscesses (single or multiple); 2) unilocular or multilocular (presence of $>1 \mathrm{~mm}$ thick septations); 3) solid or cystic appearance in most of the sections showing the cavity with abscess; 4) gas within the cavity with abscess; 5) thrombophlebitis (hypodense filling defects in the contrast-enhanced hepatic veins, their tributaries, and/ or the inferior vena cava); 6) septic pulmonary embolism 
(focal or multifocal lung infiltrates); and 7) presence of pleural effusion.

\section{Platelet reactivity}

Platelet reactivity reflected by $\mathrm{P}$-selectin expression as markers of platelet degranulation was determined by flow cytometry. ${ }^{13}$ Platelet reactivity was measured in unstimulated samples and after ex vivo platelet stimulation by ADP (low dose: $7.8 \mu \mathrm{M}$ and high dose: $31.2 \mu \mathrm{M}$; Sigma-Aldrich, $\mathrm{St}$ Louis, MO, USA). Whole blood was added to a mixture of 4-(2-hydroxyethyl)-1-piperazineethanesulfonic acid-buffered saline and saturated concentrations of PE-labeled anti-CD62P (P-selectin; BioLegend, San Diego, CA, USA) and PC7labeled anti-CD61 (platelet identification marker; Beckman Coulter, Villepinte, France). After 20 minutes of incubation at room temperature, $0.2 \%$ paraformaldehyde was added and samples were analyzed. Platelets were gated based on their forward- and sideward-scatter properties and positivity for CD61, which was defined as a median fluorescence intensity (MFI) exceeding that of its matched isotype control.

\section{PMAs}

The formation of PMA, which is considered as a sensitive marker for platelet activation, was determined by incubating citrated whole blood with PC7-labeled anti-CD61 and PElabeled anti-CD14 (a glycosylphosphatidylinositol-linked membrane glycoprotein; BioLegend) as a monocyte identification marker. ${ }^{14}$ The platelet-monocyte complex formation was quantified based on the MFI of CD61 on CD14-positive cells.

Platelet-poor plasma was harvested from citrate anticoagulated whole blood by centrifugation $(1,500 \times \mathrm{g}$ without brake, 15 minutes, $20^{\circ} \mathrm{C}$ ). The plasma concentrations of PF-4 (R\&D systems, Abbington, UK) were measured using ELISA. ${ }^{15}$

\section{Statistical analyses}

The categorical variables of patients' characteristics were compared using the chi-squared test or Fisher's exact test, as appropriate, while the continuous variables were expressed as mean values \pm SDs and compared using the Mann-Whitney $U$ test or Student's $t$-test. A multivariate logistic regression model was used to evaluate the predisposing factors of IKLAS by calculating the OR and $95 \% \mathrm{CI}$ of each clinical variable. Hosmer-Lemeshow goodness-of-fit tests were performed to evaluate the predictive performance of the logistic regression model. Differences in CT features across the groups were compared using ANOVA with posttests or chi-squared test. Data on platelet reactivity, PMA, and plasma markers levels were expressed as medians with IQR. The experimental data between multiple groups were compared using nonparametric Kruskal-Wallis test with Dunn's post test. All statistical computations were performed using SPSS Statistics version 17.0 (SPSS Inc., Chicago, IL, USA). A two-sided $P$-value of $<0.05$ was considered as statistically significant in all analyses.

\section{Results}

\section{Risk factors for invasive KP liver abscess syndrome}

Of the $175 \mathrm{DM}$ patients with community-acquired monoKP bacteremia during the study period, 41 (23.4\%) had IKLAS. Based on the results of the serotype determination by PCR assay, the K1/K2 serotype was identified in 22 (12.6\%, including $20 \mathrm{~K} 1$ and two $\mathrm{K} 2$ ) isolates. As presented in Table 1, IKLAS occurred more often in patients infected with $\mathrm{K} 1 / \mathrm{K} 2$ serotype KP strains than in those infected with non-K1/K2 serotype KP strains $(43.9 \%$ vs $3.0 \%, P<0.01)$. The proportion of IKLAS was significantly higher among DM patients with $\mathrm{HbA}_{1 \mathrm{c}} \geq 9 \%$ than among those with $\mathrm{HbA}_{1 \mathrm{c}}$ $<9.0 \%$ (78.0\% vs $54.4 \%, P=0.01$ ). In all, 24 (13.7\%) patients had received aspirin a month prior to the diagnosis of KP infection, and they were less likely to develop IKLAS than those who had not received aspirin $(2.4 \%$ vs $17.1 \%, P=0.02$; Table 1). In multivariate analysis, IKLAS developing in DM patients with community-acquired bacteremia was associated with bacteremia due to K1/K2 serotype strains (adjusted odds ratio [AOR], 8.81; 95\% CI, 2.18-35.53; $P<0.01$ ), poor glycemic control (AOR, 4.97; 95\% CI, 1.73-14.23; $P<0.01$ ), and recent treatment with aspirin (AOR, $0.17 ; 95 \%$ CI, $0.04-0.79 ; P=0.02)$. There was adequate goodness of fit (Hosmer and Lemeshow test: $\chi^{2}=0.50 ; P>0.99$ ). The receiver operating characteristic (ROC) curve analysis indicated that the predictive performance of the logistic regression model was adequate (area under the curve $[\mathrm{AUC}]=0.77$ ). Hence, there was no significant evidence of lack of fit for any of the final models.

\section{Imaging study of invasive KP liver abscess syndrome}

The characteristics of abdominal CT of 41 DM patients with different glycemic control who developed IKLAS are summarized in Table 2. A single abscess was found in 19 (46.3\%) patients and multiple abscesses in 22 (53.7\%) patients. Among the patients with multiple abscesses, the average number of lesions per patient was $2.2 \pm 0.78$. The 
Table I Comparisons of characteristics between diabetic patients with community-acquired KP bacteremia who developed invasive syndrome and those who did not develop invasive syndrome

\begin{tabular}{|c|c|c|c|}
\hline \multirow[t]{2}{*}{ Characteristics, n (\%) } & \multicolumn{2}{|c|}{ Invasive syndrome (\%) } & \multirow[t]{2}{*}{$P$-value ${ }^{a}$} \\
\hline & Yes $(n=4 I)$ & No $(n=134)$ & \\
\hline $\mathrm{K}_{1} / \mathrm{K}_{2}$ serotype of $\mathrm{KP}$ & $18(43.9)$ & $4(3.0)$ & $<0.0 \mathrm{I}^{\mathrm{b}}$ \\
\hline Male & $17(4 \mid .5)$ & $47(35.1)$ & 0.47 \\
\hline Age $\geq 60$ years & $19(46.3)$ & $65(48.5)$ & 0.86 \\
\hline Newly diagnosed diabetes & $8(19.5)$ & $15(11.2)$ & 0.19 \\
\hline $\mathrm{HbA}_{\mathrm{Ic}} \leq 7 \%$ & $4(9.8)$ & $29(21.6)$ & 0.11 \\
\hline $\mathrm{HbA}_{\mathrm{Ic}} 7 \%-9 \%$ & $5(12.2)$ & $32(23.9)$ & 0.13 \\
\hline $\mathrm{HbA}_{\mathrm{Ic}} \geq 9 \%$ & $32(78.0)$ & $73(54.4)$ & $0.01^{b}$ \\
\hline Cardiovascular diseases & $4(9.8)$ & $4(3.0)$ & 0.09 \\
\hline Liver cirrhosis & $3(7.3)$ & $5(3.7)$ & 0.39 \\
\hline Chronic renal failure & $8(19.5)$ & $21(15.7)$ & 0.64 \\
\hline Alcoholism & $3(7.3)$ & $6(4.5)$ & 0.44 \\
\hline COPD & $3(7.3)$ & $6(4.5)$ & 0.44 \\
\hline Malignancy & $3(7.3)$ & $13(9.7)$ & 0.77 \\
\hline Biliary tract diseases & I (2.4) & $4(3.0)$ & $>0.99$ \\
\hline High-dose steroid use & $3(7.3)$ & $6(4.5)$ & 0.44 \\
\hline Aspirin use & I (2.4) & $23(17.1)$ & $0.02^{\mathrm{b}}$ \\
\hline Leukocytes, $\times 10^{9} / \mathrm{L}^{c}$ & $12.2(4.4)$ & $10.4(4.4)$ & 0.75 \\
\hline Platelets, $\times 10^{9} / \mathrm{L}^{\mathrm{c}}$ & $178(45)$ & $202(57)$ & 0.87 \\
\hline C-reactive protein, $\mathrm{mg} / \mathrm{L}^{\mathrm{c}}$ & $163(97)$ & $166(85)$ & 0.83 \\
\hline
\end{tabular}

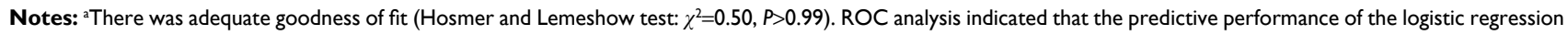
model was adequate ( $A \cup C=0.77$ ). ${ }^{b}$ Results of multivariate analysis indicated that diabetic patients with community-acquired $K P$ bacteremia who were infected with strains expressing the $\mathrm{K}_{1} / \mathrm{K}_{2}$ serotype (AOR, 8.8I; 95\% Cl, 2.18-35.53; $\left.P<0.0 \mathrm{I}\right)$ and those with $\mathrm{HbA} \mathrm{I}_{\mathrm{Ic}} \geq 9 \%$ (AOR, 4.97; 95\% Cl, I.73-I4.23; $P<0.0 \mathrm{I}$ ) were at increased risk of developing IKLAS, whereas those who had recent therapy with aspirin (AOR, $0.17 ; 95 \% \mathrm{Cl}, 0.04-0.79 ; P=0.02)$ were at a lower risk of acquiring IKLAS. ${ }^{\mathrm{C}} \mathrm{Data}$ depicted are mean values with $S D$.

Abbreviations: KP, Klebsiella pneumonia; ROC, receiver operating characteristic; AUC, area under the curve; AOR, adjusted odds ratio; IKLAS, invasive Klebsiella pneumoniae liver abscess syndrome.

Table 2 Comparisons of CT findings between diabetic patients who develop IKLAS with different $\mathrm{HbA}_{\mathrm{Ic}}$ concentrations

\begin{tabular}{|c|c|c|c|c|}
\hline \multirow[t]{2}{*}{ CT features, n (\%) } & \multicolumn{3}{|c|}{ HbA $_{\mathrm{Ic}}$ concentrations } & \multirow[t]{2}{*}{$P$-value } \\
\hline & $\leq 7 \%(n=4)$ & $7 \%-9 \%(n=5)$ & $\geq 9 \%(n=32)$ & \\
\hline Well-defined margin & $3(75.0)$ & $3(60.0)$ & $18(56.0)$ & 0.87 \\
\hline Multiple abscesses & $2(50.0)$ & $2(40.0)$ & $18(56.0)$ & 0.86 \\
\hline A cystic component & $3(75.0)$ & $4(80.0)$ & $26(81.3)$ & 0.99 \\
\hline Multilocular & $3(75.0)$ & $4(80.0)$ & $21(65.6)$ & 0.85 \\
\hline Gas in abscess & I (25.0) & $2(40.0)$ & $14(43.8)$ & 0.86 \\
\hline Hepatic venous thrombophlebitis & 0 & 0 & $15(46.8)$ & $0.03^{\mathrm{a}}$ \\
\hline Septic pulmonary embolism & I (25.0) & 0 & $10(3 \mid .3)$ & 0.40 \\
\hline Pleural effusion & $2(50.0)$ & $3(60.0)$ & $22(68.8)$ & 0.73 \\
\hline
\end{tabular}

Note: ${ }^{a}$ Diabetic patients with IKLAS in the group with poor glycemic control (47\%) had a significant probability of developing hepatic venous thrombophlebitis than those patients with suboptimal or good glycemic control $(P=0.03)$.

Abbreviations: CT, computed tomography; IKLAS, invasive Klebsiella pneumoniae liver abscess syndrome.

abscesses were predominantly solid in eight (19.5\%) patients and cystic in $33(80.5 \%)$ patients. Unilobar involvement was seen in $13(31.7 \%)$ patients, and multilocular abscess was present in $28(68.3 \%)$ patients. Gas in the abscess cavity was present in 17 (41.5\%) patients, and hepatic venous thrombophlebitis was present in $15(36.6 \%)$ patients. There were $11(26.8 \%)$ patients with septic pulmonary emboli and $27(65.9 \%)$ patients with pleural effusion. In a subgroup analysis, hepatic venous thrombophlebitis was more likely to be found in DM patients with IKLAS who had poor glycemic control than in those with suboptimal or good glycemic control $(P=0.03$; Table 2$)$. 


\section{Analysis of platelet reactivity to KP infection}

We further studied the platelet reactivity, PMAs, and soluble platelet proteins, which are sensitive markers for platelet activation, in this cohort of DM patients with communityacquired KP bacteremia. The randomly selected patient characteristics in both groups (40 patients in the IKLAS group and another 40 patients in the non-IKLAS group) were similar, including the intervals between the homeostatic testing and blood cultures performed ( $42 \pm 18$ vs $36 \pm 20$ hours, $P=0.45$ ) or antibiotic treatment initiation ( $24 \pm 8$ vs $22 \pm 12$ hours, $P=0.64$ ). The C-reactive protein levels measured when the homeostasis assays were performed were comparable between the patients in the IKLAS group and those in the non-IKLAS group ( $124 \pm 86 \mathrm{vs} 102 \pm 74 \mathrm{mg} / \mathrm{L} ; P=0.55$ ). A group of eight healthy volunteers were enrolled as controls. However, the mean age of the controls was lower than that of the patients in the IKLAS and non-IKLAS groups.

The IKLAS group had a significantly higher MFI of PMA than the non-IKLAS group and controls (Figure 1). The median MFI values (IQR) of the platelet marker CD61

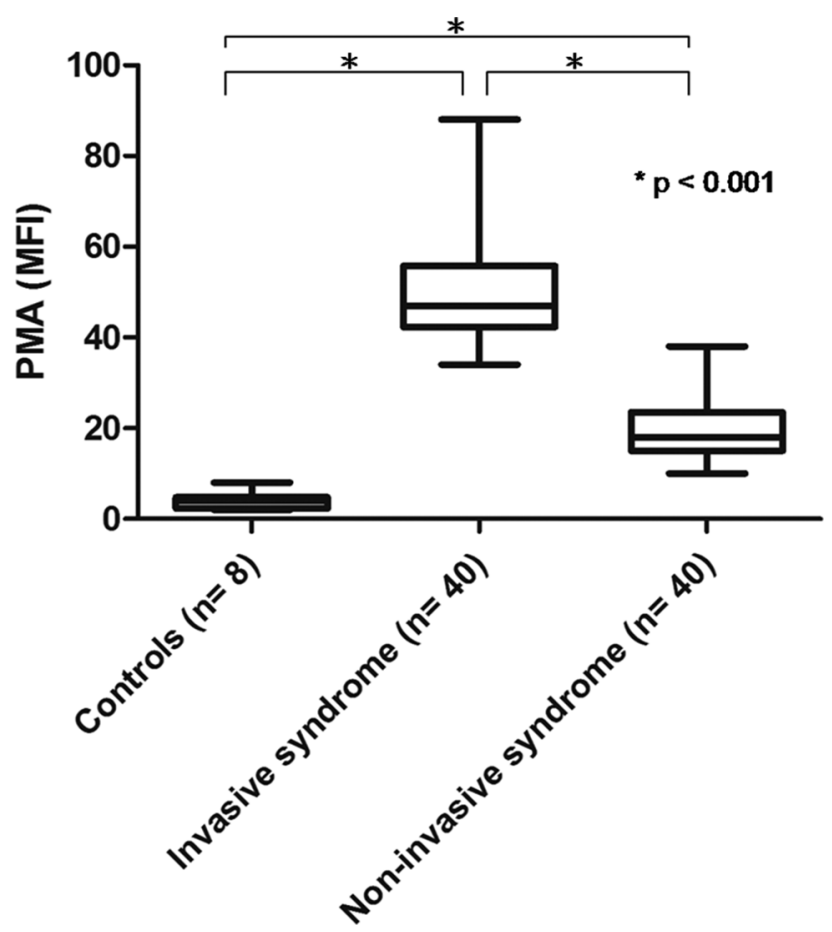

Figure I PMAs in diabetic patients with IKLAS and in those with non-IKLAS. Notes: PMAs are depicted as the MFI of the platelet marker CD6I on CDI4positive cells in healthy controls $(n=8)$, in patients with IKLAS $(n=40)$, and in patients with non-IKLAS $(n=40)$. Data depicted are medians with IQR, minimum, and maximum values.

Abbreviations: PMA, platelet-monocyte aggregate; IKLAS, invasive Klebsiella pneumoniae liver abscess syndrome; MFI, median fluorescence intensity. on CD14-positive cells were 47.0 (42.3-55.8) in the IKLAS group compared with $18.0(15.0-23.5)$ in the non-IKLAS group $(P<0.001)$ and $4.0(2.3-4.8)$ in controls $(P<0.001$; Figure 1). Moreover, patients in the IKLAS group had a significantly higher median MFI of the platelet membrane expression of P-selectin (78.0; IQR, 76.2-81.8; Figure 2) upon stimulation with a high-dose ADP than those in the non-IKLAS group (28.0; IQR, 26.0-30.0; $P<0.001)$ and controls (22.0; IQR, 18.8-23.4; $P<0.001)$. The plasma concentrations of PF-4 were statistically significantly higher in the IKLAS group than those in the non-IKLAS group and healthy controls (Figure 3); the median (IQR) concentrations of PF-4 in these respective groups were $46.0 \mathrm{ng} / \mathrm{mL}$ (32.5-57.0 ng/mL), $24.0 \mathrm{ng} / \mathrm{mL}(18.0-26.0 \mathrm{ng} / \mathrm{mL})$, and $13.0 \mathrm{ng} / \mathrm{mL}(10.5-15.5 \mathrm{ng} / \mathrm{mL})$.

We performed a subanalysis of DM patients in the IKLAS group (Figure 2) and found that those with poor glycemic control $\left(\mathrm{HbA}_{1 \mathrm{c}} \geq 9.0 \%\right)$ had a significantly higher MFI of PMA than those with suboptimal glycemic control $\left(\mathrm{HbA}_{1 \mathrm{c}}\right.$ $7 \%-9 \%)$ and good glycemic control $\left(\mathrm{HbA}_{1 \mathrm{c}}<7.0 \%\right)$. The MFI (IQR) values of PMA were $48.0(41.3-56.0)$ in the poor glycemic control subgroup, 24.0 (18.0-26.0) in the suboptimal glycemic control subgroup, and $14.0(10.5-25.8)$ in the good glycemic control subgroup (Figure 4). Aspirin treatment decreased the PMA in these tested patients. The MFI (IQR) values of PMA were $38.0(18.0-48.0)$ in patients with aspirin treatment compared with $18.0(14.3-24.0 ; P<0.001)$ in those without aspirin treatment (Figure 5). No significant correlation was observed between PMA and the time interval between hemostatic testing and the initiation of antibiotic therapy, Spearman's rank $\left(R^{2}\right)=0.07, P=0.09$.

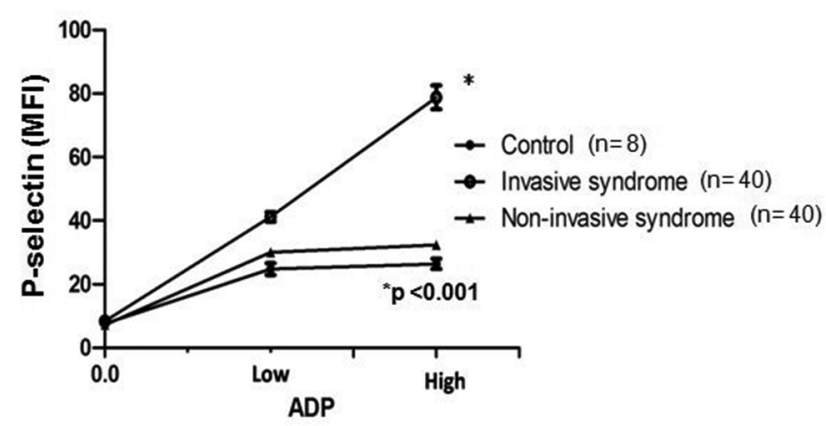

Figure 2 Platelet reactivity in diabetic patients with IKLAS and in those with nonIKLAS.

Notes: Platelet membrane expression of P-selectin is depicted as MFI in arbitrary units, at baseline and after stimulation with two concentrations of the platelet agonist ADP in healthy controls $(n=8)$, in patients with IKLAS $(n=40)$ and in patients with non-IKLAS $(n=40)$. Data depicted are median values with IQR.

Abbreviations: IKLAS, invasive Klebsiella pneumoniae liver abscess syndrome; MFI, median fluorescence intensity. 


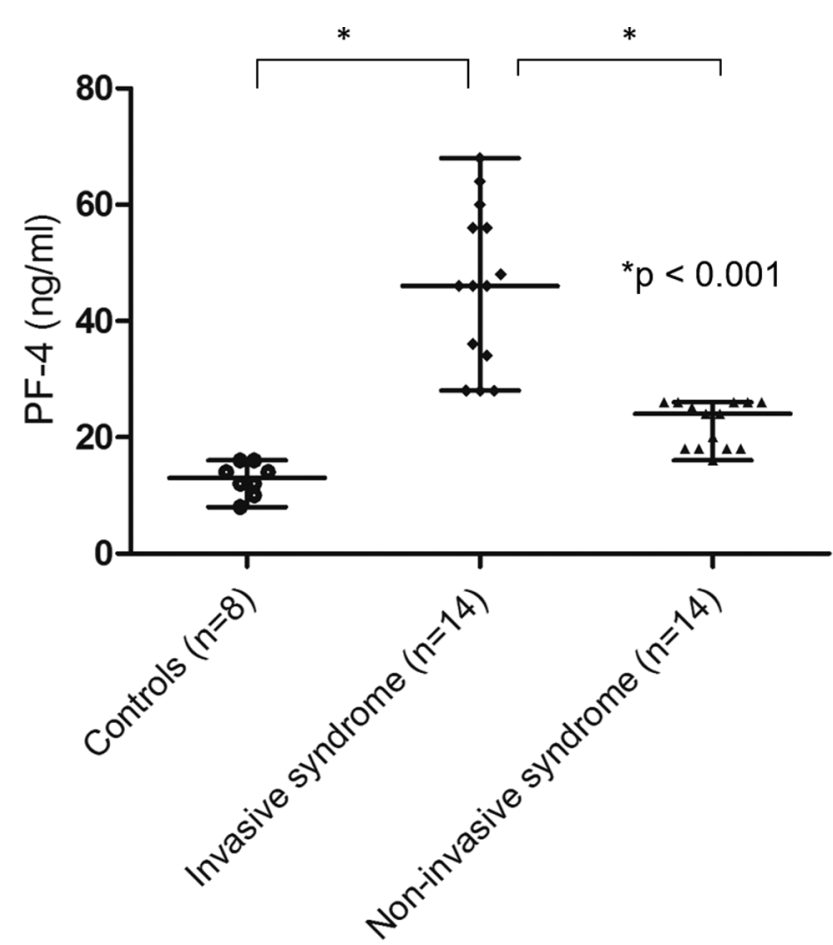

Figure 3 Plasma concentrations of PF-4 in diabetic patients with IKLAS and those with non-IKLAS.

Notes: Data from healthy controls $(n=8)$, patients with IKLAS $(n=14)$, and patients with non-IKLAS $(n=\mid 4)$. Data depicted are median values with IQR, minimum, and maximum values.

Abbreviations: IKLAS, invasive Klebsiella pneumoniae liver abscess syndrome.

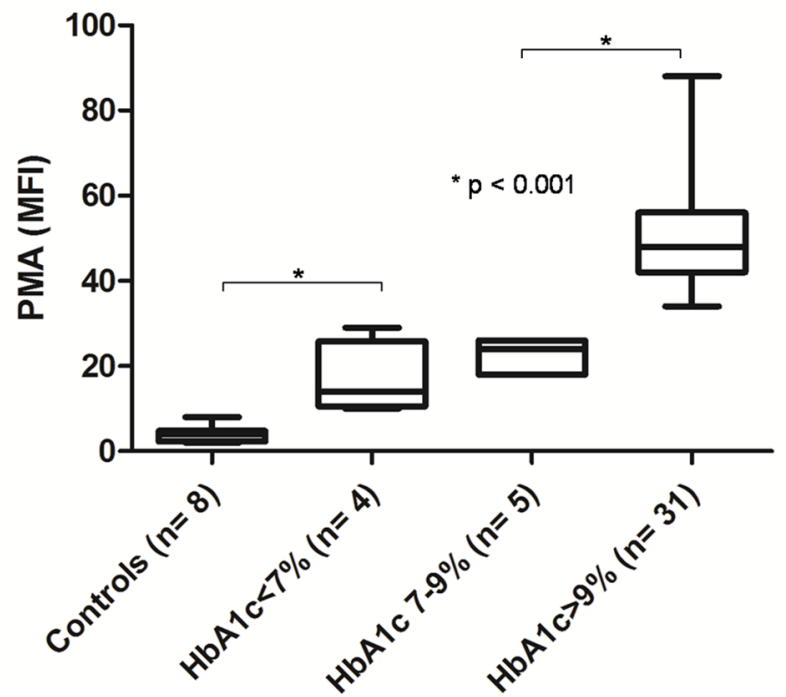

Figure 4 PMAs in diabetic patients with IKLAS, stratified with different glycemic control.

Notes: PMAs are depicted as the MFI of the platelet marker CD6I on CDI4positive cells. Data depicted are median values with IQR, minimum, and maximum values. The glycemic control was stratified into three categories: good glycemic control $\left(\mathrm{HbA}_{\mathrm{lc}} \leq 7 \%, \mathrm{n}=4\right)$, suboptimal glycemic control $\left(\mathrm{HbA}_{\mathrm{lc}} 7 \%-9 \%, n=5\right)$, and poor glycemic control $\left(\mathrm{HbA}_{\mathrm{lc}} \geq 9 \%, \mathrm{n}=3 \mathrm{I}\right)$.

Abbreviations: PMA, platelet-monocyte aggregate; IKLAS, invasive Klebsiella pneumoniae liver abscess syndrome; MFI, median fluorescence intensity.

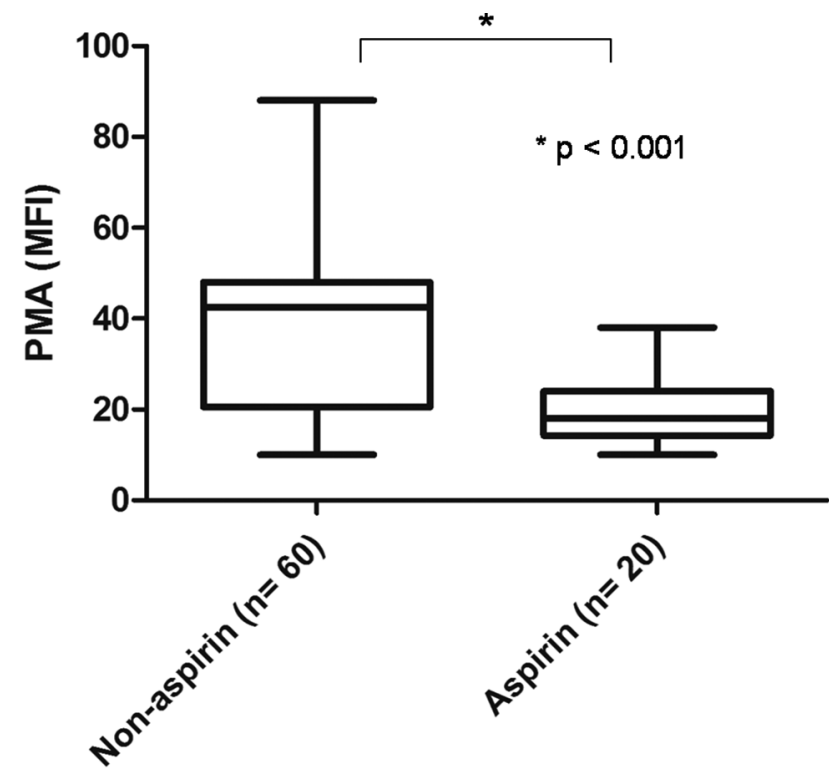

Figure 5 PMAs in aspirin and non-aspirin using diabetic patients with communityacquired KP bacteremia.

Notes: PMAs is depicted as the MFI of the platelet marker CD6I on CDI4-positive cells. Data depicted are median values with IQR, minimum, and maximum values. Abbreviations: PMA, platelet-monocyte aggregate; KP, Klebsiella pneumonia; MFI, median fluorescence intensity.

\section{Discussion}

The inflammatory response during sepsis involves both platelet and endothelial cell activation. Bacteria can either support platelet adhesion or induce platelet aggregation. ${ }^{16}$ P-selectin is a glycoprotein stored in Weibel-Palade bodies of endothelial cells or $\alpha$-granules of platelets. Upon stimulation, the expression of P-selectin increases. The surface P-selectin can be downregulated by internalization and degradation or by shedding soluble P-selectin in plasma. The activation of platelets leads to the formation of plateletleukocyte aggregates, which is both platelet and endothelial cell P-selectin dependent. ${ }^{17}$ de Stoppelaar et $\mathrm{al}^{18}$ reported that $\mathrm{P}$-selectin-deficient mice infected with KP via the airways to induce sepsis displayed 10-1,000-fold higher bacterial burdens in the lungs, blood, and distant organs, and P-selectin deficiency was associated with decreased platelet-monocyte complexes and increased cytokine release, which suggests that $\mathrm{P}$-selectin is involved in the host defense against sepsis caused by KP, with protective roles for both platelet and endothelial cell P-selectins. ${ }^{18}$ In this current study, we found that DM patients in the IKLAS group had a significantly higher PMA and platelet P-selectin expression and higher plasma PF-4 concentration than those in the non-IKLAS group and controls. The IKLAS group had a higher platelet reactivity 
than the non-IKLAS group. These differences were observed despite comparable plasma C-reactive protein concentrations, suggesting that inflammation is not a major driving force in KP-induced platelet activation.

Regional venous thrombophlebitis is a complication of various local infectious diseases that can spread throughout the body. In sepsis, microthrombi form in the blood capillaries. ${ }^{19}$ Hepatic venous thrombophlebitis frequently occurs in patients with liver abscesses and appears as non-enhancing linear structures or gas without expanding the lumen on contrast-enhanced $\mathrm{CT} .{ }^{20}$ The hepatic venous thrombophlebitis may facilitate the seeding of microorganisms into the blood circulation, which might result in hematogenous metastatic infection in patients with liver abscess caused by KP. ${ }^{21}$ Evidence suggests that DM patients have a higher platelet reactivity and baseline activation than healthy controls. ${ }^{22}$ Furthermore, a close relationship between poor glycemic control and increased platelet activity in these DM patients was noted. ${ }^{23}$ We found that DM patients with a poor glycemic control have higher rates of developing hepatic venous thrombophlebitis and invasive syndrome (Tables 1 and 2). In agreement with the previous report, ${ }^{24}$ hepatic venous thrombosis is considered as an important sign of metastatic infection in DM patients. Wang et $\mathrm{al}^{24}$ reported that hepatic venous thrombophlebitis was associated with $72.7 \%$ of metastatic infections. They also revealed that poorly controlled diabetes was associated with liver abscess caused by KP. ${ }^{24}$ In this study, we postulated that the KP might activate the platelet activity especially in DM patients with poor glycemic control and form a septic thrombosis, which can lead to seeding of microorganisms with metastatic infection.

With regard to aspirin therapy on platelet reactivity, lower soluble P-selectin levels were found in patients with stable coronary disease after a percutaneous coronary intervention under aspirin therapy. ${ }^{25}$ In addition, 1 week of low-dose aspirin therapy could attenuate platelet activation in HIV-infected adults. ${ }^{26}$ Furthermore, a well-conducted, retrospective study from the Mayo Clinic has shown significantly reduced rates of embolism in patients with infective endocarditis who had been taking aspirin prior to the diagnosis. ${ }^{27}$ These findings were also supported by a prospective investigation showing a significant reduction in the frequency of $S$. aureus bacteremia in patients with indwelling hemodialysis catheters who had been taking long-term aspirin. ${ }^{28}$ The capsular polysaccharide surrounding the KP bacteria enables the bacteria to evade phagocytosis. We found that the reduction in capsular polysaccharide by salicylates, the major metabolite of aspirin, may therefore attenuate the pathogenicity of KP stains. ${ }^{29}$ In this study, several patients used aspirin that inhibits platelet activation via the COX-1 pathway, ${ }^{30}$ leading to decreased platelet reactivity, which may contribute to lower the patients' risk for developing IKLAS.

Our study had several limitations. Peripheral blood samples were collected randomly from 80 participants after KP bacteremia. Differences among these selected participants in this approach to blood test may have resulted in ascertainment bias. The case number of the patients in each group remained small, and the hemostatic assays were only performed once in patients with positive blood cultures. KP isolated from patients in the non-IKLAS group was likely to be rapidly killed by antibiotics, despite the similar time intervals between hemostatic testing and performance of blood cultures or antibiotic treatment initiation among patients in the IKLAS and non-IKLAS groups. Still, increased platelet activation was observed among patients in the IKLAS group related to persistent bacteremia could not to be excluded in this current study. Finally, any potential contribution of other medications except aspirin taken by the patients might influence the platelet activity in the IKLAS group cannot be excluded in this study.

\section{Conclusion}

KP bacteremia complicated with IKLAS is associated with marked platelet activation, which may contribute to the occurrence of vascular complications seen in these infections. Given the role of platelets in immunity and host defense, further studies are needed to investigate the potential role of platelet inhibition in preventing the development of IKLAS in diabetic patients with KP bacteremia.

\section{Acknowledgments}

We thank Dr Chien-Ching Hung at the Department of Internal Medicine, National Taiwan University Hospital, for his critical review of this manuscript. This work was funded by grants from the Ministry of Science and Technology of Taiwan (MOST 106-2314-B-182A-137).

\section{Disclosure}

The authors report no conflicts of interest in this work.

\section{References}

1. Fang CT, Lai SY, Yi WC, Hsueh PR, Liu KL, Chang SC. Klebsiella pneumoniae genotype $\mathrm{K} 1$ : an emerging pathogen that causes septic ocular or central nervous system complications from pyogenic liver abscess. Clin Infect Dis. 2007;45(3):284-293.

2. Sobirk SK, Struve C, Jacobsson SG. Primary Klebsiella pneumoniae liver abscess with metastatic spread to lung and eye, a north-European case report of an emerging syndrome. Open Microbiol J. 2010;4:5-7. 
3. Ko WC, Paterson DL, Sagnimeni AJ, et al. Community-acquired Klebsiella pneumoniae bacteremia: global differences in clinical patterns. Emerg Infect Dis. 2002;8(2):160-166.

4. Lee SS, Chen YS, Tsai HC, et al. Predictors of septic metastatic infection and mortality among patients with Klebsiella pneumoniae liver abscess. Clin Infect Dis. 2008;47(5):642-650.

5. Fang CT, Chuang YP, Shun CT, Chang SC, Wang JT. A novel virulence gene in Klebsiella pneumoniae strains causing primary liver abscess and septic metastatic complications. J Exp Med. 2004;199(5):697-705.

6. Lee $\mathrm{CH}$, Chuah SK, Tai WC, Chang CC, Chen FJ. Delay in Human Neutrophil Constitutive Apoptosis after Infection with Klebsiella pneumoniae Serotype K1. Front Cell Infect Microbiol. 2017;7:87.

7. Timmons S, Huzoor-Akbar, Grabarek J, Kloczewiak M, Hawiger J. Mechanism of human platelet activation by endotoxic glycolipid-bearing mutant Re595 of Salmonella minnesota. Blood. 1986;68(5):1015-1023.

8. Mccabe R, Lambert L, Frazee B. Invasive Klebsiella pneumoniae infections, California, USA. Emerg Infect Dis. 2010;16(9):1490-1491.

9. Smith TL, Weyrich AS. Platelets as central mediators of systemic inflammatory responses. Thromb Res. 2011;127(5):391-394.

10. Yu VL, Hansen DS, Ko WC, et al. Virulence characteristics of Klebsiella and clinical manifestations of $K$. pneumoniae bloodstream infections. Emerg Infect Dis. 2007;13(7):986-993.

11. Selvin E, Wattanakit K, Steffes MW, Coresh J, Sharrett AR. HbA1c and peripheral arterial disease in diabetes: the Atherosclerosis Risk in Communities study. Diabetes Care. 2006;29(4):877-882.

12. Pan YJ, Fang HC, Yang HC, et al. Capsular polysaccharide synthesis regions in Klebsiella pneumoniae serotype K57 and a new capsular serotype. J Clin Microbiol. 2008;46(7):2231-2240.

13. Tunjungputri RN, Van Der Ven AJ, Schonsberg A, et al. Reduced platelet hyperreactivity and platelet-monocyte aggregation in HIVinfected individuals receiving a raltegravir-based regimen. AIDS. 2014;28(14):2091-2096.

14. Michelson AD, Barnard MR, Krueger LA, Valeri CR, Furman MI. Circulating monocyte-platelet aggregates are a more sensitive marker of in vivo platelet activation than platelet surface P-selectin: studies in baboons, human coronary intervention, and human acute myocardial infarction. Circulation. 2001;104(13):1533-1537.

15. Snoep JD, Roest M, Barendrecht AD, De Groot PG, Rosendaal FR, Van Der Bom JG. High platelet reactivity is associated with myocardial infarction in premenopausal women: a population-based case-control study. J Thromb Haemost. 2010;8(5):906-913.

16. Kerrigan SW. The expanding field of platelet-bacterial interconnections. Platelets. 2015;26(4):293-301.
17. Østerud B, Bjørklid E. Sources of Tissue Factor. Semin Thromb Hemost. 2006;32(1):11-23.

18. de Stoppelaar SF, Van't Veer C, Roelofs JJ, et al. Platelet and endothelial cell P-selectin are required for host defense against Klebsiella pneumoniaeinduced pneumosepsis. J Thromb Haemost. 2015;13(6):1128-1138.

19. Levi M, Schultz M, van der Poll T. Sepsis and thrombosis. Semin Thromb Hemost. 2013;39(5):559-566.

20. Syed MA, Kim TK, Jang HJ. Portal and hepatic vein thrombosis in liver abscess: CT findings. Eur J Radiol. 2007;61(3):513-519.

21. Maffiolo C, Novellas S, Chevallier P, Brunner P, Mourou MY, Bruneton $\mathrm{JN}$. Thrombophlebitis of the hepatic veins: complication of a Klebsiella liver abscess. Clin Imaging. 2006;30(1):63-65.

22. Kakouros N, Rade JJ, Kourliouros A, Resar JR. Platelet function in patients with diabetes mellitus: from a theoretical to a practical perspective. Int J Endocrinol. 2011;2011:742719.

23. Demirtunc R, Duman D, Basar M, Bilgi M, Teomete M, Garip T. The relationship between glycemic control and platelet activity in type 2 diabetes mellitus. J Diabetes Complications. 2009;23(2):89-94.

24. Wang HH, Tsai SH, Yu CY, et al. The association of haemoglobin $\mathrm{A}_{1} \mathrm{C}$ levels with the clinical and CT characteristics of Klebsiella pneumoniae liver abscesses in patients with diabetes mellitus. Eur Radiol. 2014;24(5):980-989.

25. Kaufmann J, Wellnhofer E, Kappert K, et al. Soluble P-selectin level correlates with acetylsalicylic acid but not with clopidogrel response in patients with stable coronary artery disease after a percutaneous coronary intervention. Coron Artery Dis. 2013;24(4):312-320.

26. O'Brien M, Montenont E, Hu L, et al. Aspirin attenuates platelet activation and immune activation in HIV-1-infected subjects on antiretroviral therapy: a pilot study. J Acquir Immune Defic Syndr. 2013;63(3):280-288.

27. Anavekar NS, Tleyjeh IM, Anavekar NS, et al. Impact of prior antiplatelet therapy on risk of embolism in infective endocarditis. Clin Infect Dis. 2007;44(9):1180-1186.

28. Sedlacek M, Gemery JM, Cheung AL, Bayer AS, Remillard BD. Aspirin treatment is associated with a significantly decreased risk of Staphylococcus aureus bacteremia in hemodialysis patients with tunneled catheters. Am J Kidney Dis. 2007;49(3):401-408.

29. Lee CH, Su LH, Liu JW, Chang CC, Chen RF, Yang KD. Aspirin enhances opsonophagocytosis and is associated to a lower risk for Klebsiella pneumoniae invasive syndrome. BMC Infect Dis. 2014;14:47.

30. Schrör K. Aspirin and platelets: the antiplatelet action of aspirin and its role in thrombosis treatment and prophylaxis. Semin Thromb Hemost. 1997;23(4):349-356
Infection and Drug Resistance

\section{Publish your work in this journal}

Infection and Drug Resistance is an international, peer-reviewed openaccess journal that focuses on the optimal treatment of infection (bacterial, fungal and viral) and the development and institution of preventive strategies to minimize the development and spread of resistance. The journal is specifically concerned with the epidemiology of antibiotic

\section{Dovepress}

resistance and the mechanisms of resistance development and diffusion in both hospitals and the community. The manuscript management system is completely online and includes a very quick and fair peerreview system, which is all easy to use. Visit http://www.dovepress.com/ testimonials.php to read real quotes from published authors. 